\title{
Sleep Apnea Patients Monitoring Using Mobile Phone
}

\author{
Nilam Maske, Dr. A. N. Gaikwad \\ ${ }^{1,2}$ Zeal College of Engineering and Research, Sr.No 39, Off Mumbai-Bangalore Express Highway, Narhe, Pune
}

\begin{abstract}
Real-time Obstructive Sleep Apnea (OSA) episode detection and monitoring are important for society in terms of an improvement in the health of the general population and of a reduction in mortality and healthcare costs. Currently, to diagnose OSA patients undergo PolySomnoGraphy (PSG), a complicated and invasive test to be performed in a specialized center involving many sensors and wires. Accordingly, each patient is required to stay in the same position throughout the duration of one night, thus restricting their movements. This paper proposes an easy, cheap, and portable approach for the monitoring of patients with OSA. It is easy to perform from the patient's point of view because only three wearable sensor is required, so the patient is not restricted to keeping the same position all night long, and the detection and monitoring can be carried out in any place through the use of a mobile device.
\end{abstract}

Keywords: Sleep apnea, GSM, PSG, smart phone, Wearable sensors, Real time monitoring system

\section{Introduction}

Sleep apnea is a sleep disorder characterized by the repetitive reduction of airflow during sleep, which in turn causes pauses and reduced breathing. These recurring arousals from sleep due to a blockage of airway cause fragmented sleeping patterns and lead to the activation of the body"s sympathetic nervous system. An apnea is defined as the duration of time when there is complete blockage of airflow for 10 seconds or more, and is measured in apnea-hypopnoea index (AHI) [1].

Sleep apnea (SA) in the form of Obstructive sleep apnea (OSA) is becoming the most common respiratory disorder during sleep, which is characterized by cessations of airflow to the lungs. These cessations in breathing must last more than 10 seconds to be considered an apnea event. Apnea events may occur 5 to 30 times an hour and may occur up to four hundred times per night in those with severe SA [2].

The most frequent night symptoms of SA can include snoring, nocturnal arousals, sweating and restless sleep. Moreover, like all sleeping disorders, symptoms of sleep apnea do not occur just during the night. Daytime symptoms can range from morning headaches, depression, impaired concentration and excessive sleepiness which cause mortality from traffic and industrial accidents. However, these symptoms are not definitive to detect SA syndrome [3] [4].

In fact, SA is not a problem to be taken lightly, since it is associated with a major risk factor of health implications and increased cardiovascular disease and sudden death. It has been linked to irritability, depression, sexual dysfunction, high blood pressure (hypertension), learning and memory difficulties, in addition to stroke and heart attack [3][4].Several treatments options for OSA patients include weight loss, positional therapy, oral appliances, surgical procedures and continuous positive airway pressure (CPAP). CPAP is a common and effective treatment especially for patients with moderate to severe OSA. CPAP devices are masks worn during sleep that improves oxygen saturation and reduces sleep fragmentation [5].
According to the World Health Organization approximately 100 million people worldwide have obstructive sleep apnea (OSA). In the United States, OSA is estimated to affect 1 in 4 men and 1 in 9 women; it also affects 23 million working adults [5]. Approximately $4 \%$ of men and $2 \%$ of women over the age of 35 years have symptomatic moderate or severe OSA, affecting approximately 12 million people in the United States. It is estimated that less than $25 \%$ of OSA sufferers have been diagnosed [6].

The diagnosis of OSA can be done through a number of different tests and techniques. However, the symptoms of the OSA disorder are not very specific and may sometimes be caused by factors other than sleep apnea such as alcohol intake, lack of sleep and stress. Therefore, sleep specialists need to perform appropriate tests to rule out and distinguish these symptoms. If these results suggest sleep apnea, then further diagnostic tests need to be performed, which include medical, physical and sleep history exams [7].

Once the probability of OSA is determined to be high from the conducted tests, then a polysomnography is usually conducted. Polysomnography is the most common sleep study that is performed overnight and measures the patient's brain activity along with a number of other measurements. Polysomnography (PSG) is a complicated procedure and certain way of assessing the OSA problem. This is due to several reasons, including first, the inconvenience since it requires the patient to be connected to numerous sensors and to stay in hospital for one night. Second, it is expensive. The average cost for a PSG is $\$ 2,625$ due to the need for the study to take place in a specially equipped medical facility, in addition to the requirement of having a sleep lab staff overnight, trained in ,scoring ${ }^{\text {ee }}$ the resultant measurements manually. Third, along wait list of up to 6 months is caused by limited availability of PSG . 


\section{International Journal of Science and Research (IJSR) \\ ISSN (Online): 2319-7064}

Index Copernicus Value (2013): 6.14 | Impact Factor (2014): 5.611

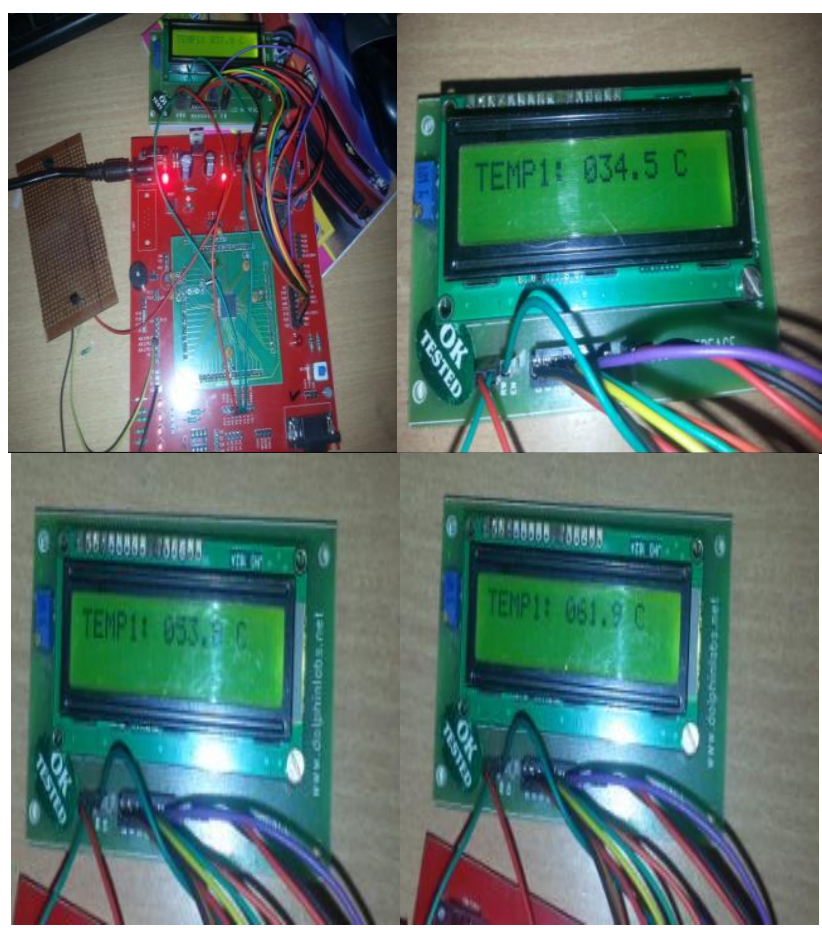

Figure 1: Temperature sensor model of Sleep Apnea

In this temperature sensor model, $16 \times 2$ lcd display is used.In this display, 1 and 3 pin of LCD connect to ground, 2 pin connected to the supply voltage. The data pin connected to the controller port $\mathrm{P} 0.8$ to $\mathrm{P} 0.15$. The $\mathrm{RS}, \mathrm{R} / \mathrm{W}, \mathrm{EN}$ pin connected to controller port $\mathrm{P} 0.2$ to $\mathrm{P} 0.4$,pin 15 connected to supply voltage and pin 16 connected to the ground.In LM35 temperature sensor,Pin 1 is connected to the supply voltage , pin 2 connected to the controller port P0.29 and Pin 3 connected to the ground. The $1 \mathrm{~K}$ ohm resistor is attached between the pin 2 and pin 3 for current limiting.

Our research study aims to develop a simpler system that provides a reliable, inexpensive, and faster approach to assessing OSA in patients before, during and after medical treatments.

\section{Literature Survey}

Over the fast few year most of the related research has focused on presenting methods for the automatic processing of different statistical features of different signal such as thorax and abdomen effort signal, nasal air flow, oxygen saturation, electrical activity of the heart(ECG), and electrical activity of the brain(EEG) for the detection of the SA[8-10].

First, in case of [8] It could reduce research time and improve medical service efficiency regarding Obstructive Sleep Apnea (OSA) detection systems. Much of the past and the current apnea research, the vital signals features and parameters of the SA automatic detection are introduced. The applications for the earlier proposed systems and the related work on real-time and continuous monitoring of OSA and the analysis is given. The study concludes with an assessment of the current technologies highlighting their weaknesses and strengths which can set a roadmap for researchers and clinicians in this rapidly developing field of study.
Second in case [9] Obstructive Sleep Apnea (OSA) is a sleeping disorder characterized by the repetitive reduction of airflow during sleep. In this paper, it consist the design and implementation of a user-friendly mobile application developed on multiple platforms to monitor and detect symptoms of sleep apnea using the smart phonees built-in sensors. The purpose of the application, Sleep Apnea Monitor (SAM), is to allow users to get a sense of whether or not they are likely to have sleep apnea, before continuing with more expensive and advanced sleep tests. In addition, SAM provides doctors and sleep specialists with remote access to patients ${ }^{\text {ee }}$ records and allows them to confirm their initial diagnosis. The parameters measured by this application are breathing patterns and movement patterns, which are recorded respectively using the built-in microphone and accelerometer. The recorded data is sent to a server for analysis in order to diagnose patients and maintain geographical studies of areas with sleep apnea patterns. The application is successfully tested among a number of users in the UAE. The system diagnoses and reports the level of the user"s sleep apnea. In addition, doctors can remotely monitor users through the website, which is interfaced with Google Maps to keep track of user locations, and keep track of their analyzed records.

[10] it consist a low-cost, real-time sleep apnea monitoring system 'AApnea Med Assist” for recognizing obstructive sleep apnea episodes with a high degree of accuracy for both home and clinical care applications. The fully automated system uses patient's single channel nocturnal ECG to extract feature sets, and uses the support vector classifier (SVC) to detect apnea episodes. "Apnea Med Assist" is implemented on Android operating system (OS) based smart phones, uses either the general adult subject-independent SVC model or subject-dependent SVC model, and achieves a classification $F$-measure of $90 \%$ and a sensitivity of $96 \%$ for the subjectindependent SVC. The real-time capability comes from the use of 1-min segments of ECG epochs for feature extraction and classification. The reduced complexity of "Apnea Med Assist" comes from efficient optimization of the ECG processing, and use of techniques to reduce SVC model complexity by reducing the dimension of feature set from ECG and ECG-derived respiration signals and by reducing the number of support vectors.

In fast few year however, statistic shows that around 100 million people worldwide, where in the US form 18 to 50 million people, are suspected to have OSA, of which more than $80 \%$ undiagnosed [11]. The trouble of having examinations discourages patient prone to OSA (Obstructive Sleep Apnea) undergo at overnight clinical research through polysomnographic data. Polysomnography (PSG) is a complicated procedure and certain way of accessing OSA problem. Complete PSG include the monitoring of breath airflow, respiratory movement, oxygen saturation $\left(\mathrm{SpO}_{2}\right)$, body position, electroencephalography (EEG), electromyography (EMG), electrooculography (EOG), and electrocardiography (ECG) [12].

[12] Obstructive sleep apnea (OSA) is the most common form of different types of sleep-related breathing disorders. It is characterized by repetitive cessations of respiratory flow

\section{Volume 5 Issue 1 January 2016}




\section{International Journal of Science and Research (IJSR) \\ ISSN (Online): 2319-7064}

Index Copernicus Value (2013): 6.14 | Impact Factor (2014): 5.611

during sleep, which occurs due to a collapse of the upper respiratory airway. OSA is majorly undiagnosed due to the inconvenient Polysomnography (PSG) testing procedure at sleep labs. This paper introduces an automated approach towards identifying the presence of sleep apnea based on the acoustic signal of respiration.

\section{Existing System}

The detection and monitoring of OSA without making use of examination carried out through the use of polysomnography is a problem that can in general be approached from several possible viewpoints. including first, the inconvenience since it requires the patient to be connected to numerous sensors and to stay in hospital for one night. Second, it is expensive. The average cost for a PSG is $\$ 2,625$ due to the need for the study to take place in a specially equipped medical facility, in addition to the requirement of having a sleep lab staff overnight, trained in ,scoring "e the resultant measurements manually. Third, along wait list of up to 6 months is caused by limited availability of PSG.

In this paper, we investigate the different current apnea researches which provide an alternative to the expensive PSG visual scoring method which is commonly used today to assess a patient' s sleep quality. In oldest result I still require improvement, therefore, in this circuit is needed to eliminate EEG signal artifacts and enable the system can recognize SA (Sleep Apnea) episode

\section{Disadvantages of Existing System}

- Polysomnography require minimum of 22 wire attachment to the patients in a specialized sleep labooatory.

- Polysomnography reuire to stay in hospital for one night and also stay at one position.

\section{System Architecture}

Propose Architecture of our system shown below in figure. It consist three sensors that is oral and nasal sensors, Temperature sensor, Heart beat sensor. The oral and nasal sensor can be used for airflow and it is place below the nose and above the upper lips. Temperature sensor (LM35) can be used for temperature measurement and it consist the +10.0 $\mathrm{mV} /{ }^{\circ} \mathrm{C}$ scale factor. In Heart beat sensor, Heart rate is the number of Heartbeats_per unit of time, typically expressed as beats per minutes (bpm). Heart rate can vary as the body's need to absorb oxygen and excrete carbon dioxide changes, such as during exercise or sleep.

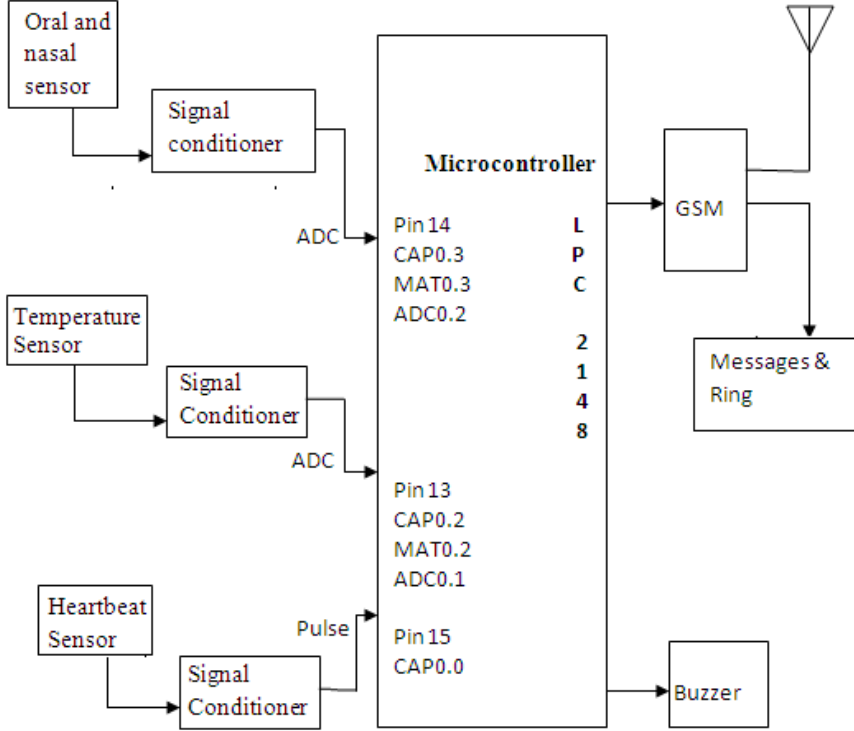

Figure 2: Block diagram of Propose Architecture

The working of this basic model is as follows. The patient is required to attach the data gathering module to the body using wearable straps etc. provided with the module. The module (consisting of medical sensors) will record the patient's data and forward it to the microcontroller. The microcontroller will process the data, display the current reading on the LCD, then to perform analysis of data and records the readings in the patient's history.

Microcontroller will compare the measured value with the predefined values recommended by the respective doctor and send it to the doctor through GSM. In case if any of the measured parameter exceeds the predefined values of the respective parameter then the system would blow the buzzer automatically and alerts all the near and dear once.

At the same time it sends the emergency message to the patient's doctor who is supposed to send the acknowledgement after reading that message. If the microcontroller does not get the acknowledgement within a specific duration of time then it sends the same emergency message to the $2^{\text {nd }}$ prior doctor. This way the immediate action could be taken to provide necessary help to the patient.

\section{Application}

- As the device is portable, small and cheap, it is beneficial in ambulance, old age home etc.

- Heart or blood pressure monitors for patients receiving cardiac care.

- It has ability for the care provider to view stored reading from the computer.

Immediate response from the doctor is possible as GSM facility is provided

\section{Conclusion}

SAM is a mobile application designed to remotely monitor the sleeping patterns of individuals and assess their likeliness 


\section{International Journal of Science and Research (IJSR) \\ ISSN (Online): 2319-7064}

Index Copernicus Value (2013): 6.14 | Impact Factor (2014): 5.611

to have symptoms of sleep apnea. Even though mobile applications that monitor sleep apnea exist, these applications only monitor one aspect (motion, snoring pattern or oxygen level) as a stand-alone project. However, this work is different and innovative in the sense that it combines the different aspects (motion and voice recorder) into one system in order to provide a preliminary diagnosis of user's sleeping pattern anywhere at any time.

This application is targeted at any individual who suspects a sleeping disorder, regardless of his/her age. By using this application, the user can monitor his/her sleep at a very lowcost from the convenience of his/her home, compared to the expensive polysomnographic tests, which require the user to visit a specialized hospital and spend a night in it wearing uncomfortable monitoring equipment.

The system also has a location tracking feature using GSM cell tower triangulation that allows authorized doctors to view locations of the users as well as the severity of their condition. This feature can be customized and markers can be plotted according to gender, age group, employment as well as other factors for research purposes and increase awareness of this growing problem.

\section{Acknowledgement}

I would like to thank the anonymous referees for their helpful guidance that has improved the quality of this paper. Also I would like to thank my Project Guide Dr.A.N.Gaikwad, for his valuable guidance.

\section{References}

[1] A. Malhotra, and D. White, "Obstructive Sleep Apnea," in The Lancet, 360(9328), 237-245,July 2002

[2] Sleep Disorders Guide. www.sleepdisorderguide.com.

[3] N. Israel, A. Tarasiuk, and Y. Zigel,"Nocturnal Sound Analysis for the Diagnosis of Obstructive Sleep Apnea," in Proceedings of the 32nd IEEE International Conference on Engineering in Medicine and Biology Society (EMBS 2010), pp. 6146-6149, Sep. 2010.

[4] A. Yilmaz, and T. Dundar, "Home Recording for PrePhase Sleep Apnea Diagnosis by Holter Recorder Using MMC Memory," in Proceedings of the 2010 IEEE International Conference on Virtual Environments Human-Computer Interfaces and Measurement Systems (VECIMS), pp.126-129, Sep. 2010.

[5] Choosing a CPAP," American Sleep Apnea Association, www.sleepapnea.org/resources/pubs/cpap/htm.

[6] "Obstructive Sleep Apnea FAQs," Apnex Medical Inc.

[7] M. Ahmed, N. Patel and I. Rosen, "Portable monitors in the diagnosis of obstructive sleep apnea," in Chest, 132(5), 1672-1677, 2007.

[8] A Panoramic Study of Obstructive Sleep Apnea Detection Technologies Laiali Almazaydeh, Khaled Elleithy and Miad Faezipour Department of Computer Science and Engineering University of Bridgeport Bridgeport, CT 06604, USA

[9] Sleep Apnea Monitoring Using Mobile Phones Shamma Alqassim, Madhumeta Ganesh, Shaheen Khoja, Meher Zaidi, Fadi Aloul, Assim SagahyroonDepartment of
Computer Science \& Engineering, American University of Sharjah, UAE

[10]Apnea MedAssist: Real-time Sleep Apnea Monitor Using Single-Lead ECGMajdi Bsoul, Member, IEEE, Hlaing Minn, Senior Member, IEEE, and Lakshman Tamil, Senior Member, IEEE

[11] SleepMedInc. www.sleepmed.md

[12]D. Baraglia et al.,"Automated Sleep Scoring and Sleep Apnea Detection in Children," in Proceedings of SPIE 6039, 2005.

\section{Author Profile}

Nilam S. Maske received his B.E. degree in Electronic and Telecommunication Engineering from Abhinav College of Engineering Shivaji University, Bhor, Pune in June 2013.

Dr. A. N. Gaikwad received his B.E in Electronics \& Telecommunication Engineering from COEP-Govt. College of Engineering, university of pune in year 1983. He received M.E. in VLSI \& Embedded System from COEP-Govt. college Pune. He completed his $\mathrm{PhD}$ in Biometric-IP for improved performance of higher order Systems in the year 2004. He worked as Lecturer at Shri Tuljabhavani college of Engineering Aurangabad for 15 year during Sept.1983 to Aug.1997. He Worked as Assistant Professor at Shri Tuljabhavani college of Engineering Aurangabad \& VIT College, Pune for 9 year during Sept.1997 to June 2005. He has 3 years of Professor Experience in VIT College, pune from July 2005 to Jan.2007. He worked as Principal at Marathwad Mitra Mandales College of Engineering,pune \& PICT college,pune for 6 year during Feb.2007 to Aug.2012. Since $1^{\text {st }}$ Sept.2012, He is working as a Principal at Zeal Education Society"s Zeal (formerly Dnyanganga) College of Engineering \& Research, Pune. 